\title{
Gestión de procesos operativos del observatorio de ciencias empresariales de la universidad de otavalo
}

\section{Operational process management of the observatory of business science of the university of otavalo}

\author{
DOI: $10.46932 / \operatorname{sjdv} 3 n 1-051$
}

Received in: Jan 30st, 2021

Accepted in: Feb 1th, 2022

\author{
Edwin Santiago Núñez Naranjo \\ Msc. \\ Universidad de Otavalo, Imbabura-Ecuador \\ E-mail: snunez@uotavalo.edu.ec \\ Belky Nerea Orbes Revelo \\ Msc. \\ Universidad de Otavalo, Imbabura-Ecuador \\ E-mail: borbes@uotavalo.edu.ec
}

\section{RESUMEN}

La investigación tuvo por objetivo realizar una propuesta de gestión para los procesos operativos del Observatorio de Ciencias Empresariales de la Universidad de Otavalo con sus siglas (OBCE). Dicha propuesta incluye cuatro procesos: inteligencia comercial, comercio exterior, relaciones internacionales e inversión extranjera, todos ellos con sus respectivos procedimientos. Como respaldo teórico a la propuesta se presentaron los postulados administrativos de gestión por procesos desarrollados por autores institucionales como la Universidad de Nariño y también personales como Martin Álvarez. El documento se elaboró a partir de una metodología de investigación mixta, compuesta por instrumentos de levantamiento de información cuantitativa (encuesta) y cualitativita (entrevista), con el fin de poder realizar un diagnóstico situacional de la forma en que los cuatro procesos atenderán las necesidades de los micro, pequeñas y medianas empresas de la sierra norte ecuatoriana que tienen potenciales productos de exportación o requieren inversión extranjera para poder internacionalizarse. Para la propuesta de gestión de los procesos operativos del observatorio, se contempló la realización de flujogramas que representan de forma práctica la planificación de los procesos con sus respectivos procedimientos y resultados que una vez creado el observatorio se ejecutarán y conformarán sus pilares. En el trabajo se concluye que los cuatro procesos operativos son los generadores de valor agregado de dicho centro de investigaciones, ya que aportan directamente con su operación, tanto en la investigación y publicación constante, así como en la asesoría y apoyo a los emprendedores ecuatorianos en comercio exterior e inversión extranjera.

Palabras clave: procesos operativos, inteligencia comercial, comercio exterior, relaciones internacionales, inversión extranjera, MIPYMES.

\begin{abstract}
The objective of the research was to make a management proposal for the operational processes of the Observatory of Business Sciences of the University of Otavalo (known as OBCE). This proposal includes four processes: commercial intelligence, international trade, international relations and foreign investment, all of them with their respective procedures. As theoretical support to the proposal, administrative management postulates were presented by processes developed by institutional authors
\end{abstract}


such as the University of Nariño as well as personal authors such as Martin Álvarez. The document was based on a mixed research methodology, composed of quantitative (survey) and qualitative (interview) paradigms, which will be used to make a situational diagnosis of the way in which the four processes will address the needs of micro, small and medium enterprises in the Ecuadorian northern highlands that have export products or require foreign investment to internationalize themselves. This proposal included the creation of flowcharts that represent in a practical way the planning of the processes with their respective procedures and results that once created the observatory will be executed. The work concluded that the four operational processes are the generators of added value of this research center, since they contribute directly with their operation: in constant research and publication, as well as in technical support of Ecuadorian entrepreneurs in international trade and foreign investment.

Keywords: operative processes, commercial intelligence, international commerce, international commerce, foreign investment, Small and Medium Enterprises (MSMEs).

\section{INTRODUCCIÓN}

"Los observatorios económicos tienen su origen en Europa hacia finales de la década de los ochenta" (Phélan, 2007), a pesar de que se ha difundido su importancia a todas partes del mundo. Desrosières (1996) afirma que "fueron creados hacia la década de los sesenta en Francia por el Institut National de la Statistique et des Études Économiques)" a partir de la constatación de una insuficiencia de conocimientos técnicos en diversas áreas de la economía y administración. De ahí que se originó la necesidad de crear diversos observatorios a nivel mundial, que estén destinados a diversos ámbitos de aplicación. Angulo (2009), como citó a Prieto (2003), afirma que "todo observatorio busca propósitos elementales: investigar, revisar, describir, evaluar, discutir, cuestionar, sugerir. Los contenidos que aparecen en el espacio de observación pertinente a su área de interés; e informar a la comunidad los hallazgos que ocurren en ese proceso “ (pág. 8). Por este motivo se han creado diversos tipos de observatorios los cuales son destinados a actividades económicas, de comercio e innovación, que ayuda a micro, pequeñas y medianas empresas.

En América Latina, los observatorios de comercio son escasos. Los 12 existentes en esta materia se han creado con el proposito de generar información cientifica y presentarla a través de boletines o publicaciones, aunque más de la mitad carecen de mediciones económicas en el ámbito del comercio exterior. A continuación se presentan varios casos: "RED OSEL" de Perú, Observatorio de Comercio Internacional (OCI) cuya información está asociada a los campos: social, cultural, en México el Universitario de las Innovaciones, "un espacio multidimensional constituido por redes temáticas colaborativas que recogen información sobre procesos innovadores internos y externos" (Angulo, 2009, pág. 8). En Colombia, se han creado dos Observatorios de Comercio Exterior, el Observatorio de Comercio Exterior de la Universidad Católica de Oriente, y el Observatorio Virtual de Asia Pacífico, de la Universidad Jorge Tadeo Lozano en Bogotá (Duque, 2013). 
En la sierra ecuatoriana, existen dos observatorios económicos que no abordan temas de comercio exterior propiamente, tales como: el Observatorio de la Pequeña y Mediana Empresa que está vinculado a la "Universidad Andina Simón Bolívar" y el Observatorio de la Empresa Municipal CONQUITO (CONQUITO, 2017, pág. 1). Por lo tanto, la Universidad de Otavalo ha planteado la creación del Observatorio de Comercio Exterior e Inversión Extranjera para beneficio en primera instancia de la zona norte del Ecuador.

En este contexto, se dará a conocer en la presente investigación en primer lugar los procesos (inteligencia comercial, comercio exterior, relaciones internacionales e inversión extranjera) que conforman la función sustantiva del centro de investigaciones y posteriormente se realizará una descripción de cada uno de ellos con sus respectivos procedimientos representados en flujogramas. A continuación, se presenta una visión general de dichos procesos.

La Inteligencia Comercial procurará la correcta interacción entre los usuarios del observatorio con los demás procesos operativos, considerando que es la puerta de entrada de los requerimientos de la ciudadanía y el proceso encargado de gestionar una correcta respuesta a los mismos para una mejor toma de decisiones por parte del usuario (Cano, 2015, pág. 66).

Por su parte, las Relaciones Internacionales son vínculos que se establecen entre organizaciones o empresas que buscan tener acuerdos que los beneficie, por medio de políticas comerciales, acuerdos, solución de problemas, relaciones diplomáticas, optimizar las relaciones exteriores con miras a mejorar el comercio teniendo como fin vincular a los nuevos usuarios del Observatorio con nuevos mercados.

La inversión extranjera es un fenómeno que se ha caracterizado por un gran dinamismo, consecuencia del cambio que han experimentado las relaciones económicas internacionales en las primeras dos décadas del siglo XXI. En términos generales, este proceso dentro del observatorio tiene la finalidad de servir como una guía sistemática para la captación eficaz de inversión extranjera y a su vez de inversión nacional hacia el extranjero. Para ello, el proceso de inversión extranjera busca generar información técnica y relevante sobre los flujos de inversión proveniente y saliente hacia mercados internacionales, identificar mecanismos de atracción de inversión extranjera a partir de la investigación y canalizarla de forma efectiva.

\section{METODOLOGÍA EMPLEADA Y MATERIALES UTILIZADOS}

Para la presente investigación se usará tres tipos de estudio: el descriptivo, exploratorio, y proyectivo, debido a que se busca proponer un proyecto y especificar los principales aspectos que influyen en el establecimiento de los procesos operativos del Observatorio de Ciencias Empresariales de la 
Universidad de Otavalo y evitar el manejo empírico de sus procesos y procedimientos derivados en la institución (Hernández, Fernández y Baptista, 2010).

\section{ANÁLISIS}

La tarea actual de la administración es interpretar los objetivos propuestos que plantean las organizaciones y estructurarlos a través de procesos y procedimientos que "se entienden como los lineamientos que orientan el establecimiento de pautas para normalizar y definir la operación y administración de una organización” (Álvarez, 2014, pág. 28).

Así es como el Observatorio de Ciencias Empresariales de la Universidad Otavalo ha creado áreas que ayuden a transformar en acción organizacional por medio de procesos y procedimientos sus acciones. Para ello, se define a los procesos como "un conjunto de las fases sucesivas de un fenómeno natural o de una operación artificial", los que abarcan comportamientos de la empresa que sirven para diseñar mejoras, y se defina a los procedimientos como métodos de ejecucion (Real Academia Española, 2018).

El proceso y el procedimiento entonces permite que una empresa funcione de manera correcta, debido a que estos establecen claramente los estamentos, políticas, normas, reglamentos y todo aquello concerniente a la gestión de las actividades de una institucion. Para el OBCE, se ha definido cuatro procesos, correspondientes a las siguientes áreas: inteligencia comercial, comercio exterior, relaciones internacionales e inversión extranjera, cuyos procedimientos se describen en las siguientes líneas.

\section{RESULTADOS Y DISCUSIÓN}

\subsection{INTELIGENCIA COMERCIAL.}

El proceso de Inteligencia Comercial está constituido por cuatro procedimientos: recepción, almacenamiento, investigación, evaluación y control.

Recepción: Este proceso inicia con la recepción del requerimiento realizado por el usuario, seguido a esto se realiza la determinación de las áreas operativas, a las cuales se les remite el requerimiento para su correspondiente análisis, evaluación y respuesta.

Almacenamiento: Después de que la información es revisada por el área operativa, se procede a entregar al cliente una solución a su solicitud y se crea el expediente del usuario que será archivado en las bases de datos del observatorio.

Investigación: Este procedimiento es independiente a los dos anteriores. En esta etapa del proceso se realiza las investigaciones de mercados internacionales con el fin de realizar boletines y publicaciones periódicas respecto de los resultados obtenidos para la internacionalización de productos y servicios de las micro, pequeñas, medianas empresas de la zona norte del Ecuador. 
Evaluación y Control de Resultados: Una vez entregados los resultados de asesoramiento o investigación, se evalúa y se ejecuta la valoración sobre el nivel de satisfacción, por último, se analiza y supervisa las áreas que se encontraron involucradas en las actividades (Ver gráfico 1).

Gráfico No.1 Flujograma del Proceso de Inteligencia Comercial en el OBCE

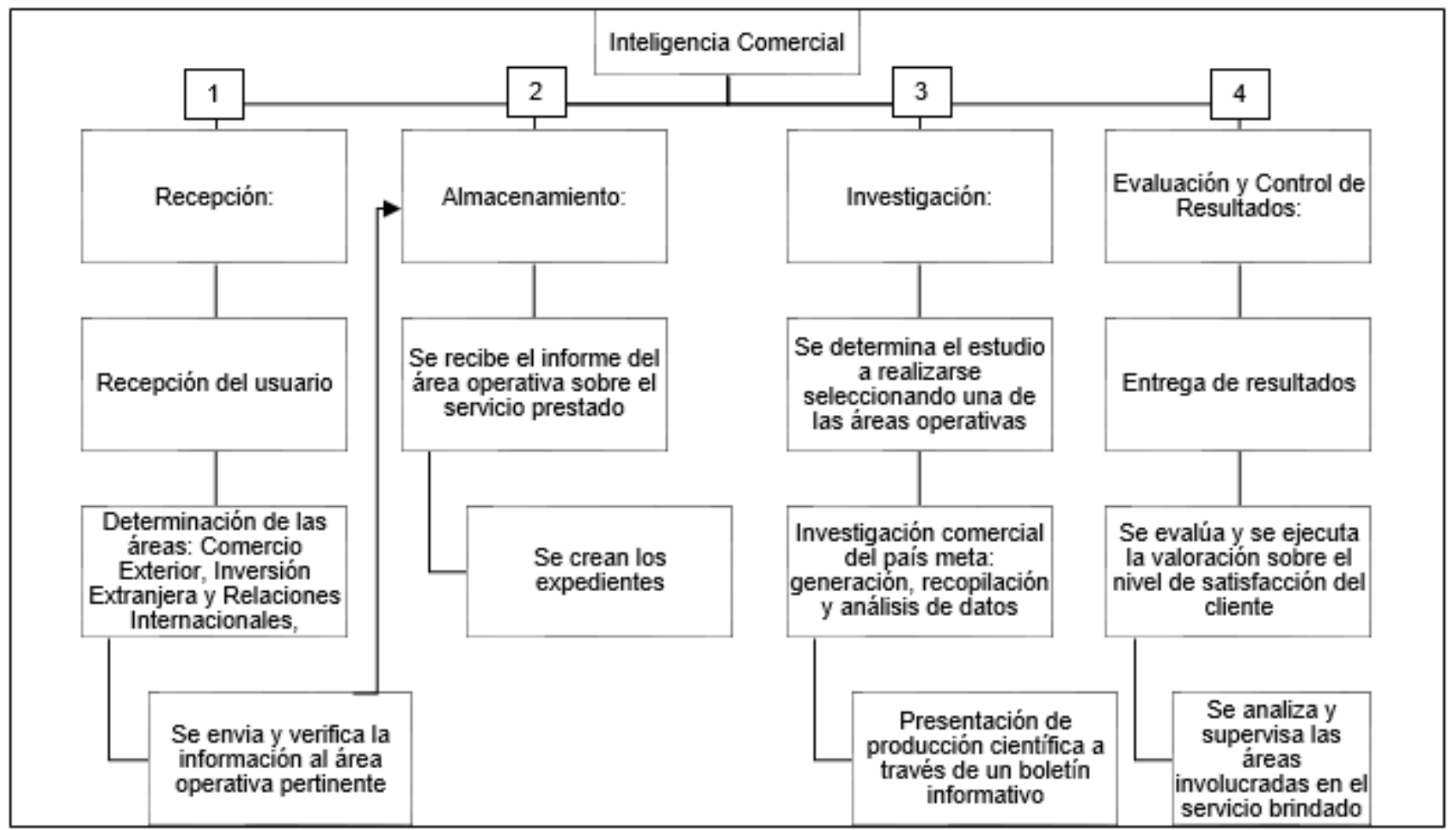

Elaborado por: Los autores

\subsection{COMERCIO EXTERIOR}

Los procedimientos que conforman el proceso de comercio exterior son dos: importación y exportación. En el primer caso, el departamento se encarga de realizar una revisión de la normativa nacional de importaciones que incluye el tema documental, logístico y aduanero dependiendo del producto. En el segundo caso, el departamento se encarga de realizar una investigación del mercado internacional con base en los requisitos arancelarios y no arancelarios para su internacionalización; y finalmente se ejecuta una revisión documental previo a la exportación. Las actividades mencionadas se presentan en el siguiente flujograma (Ver gráfico 2). 
Gráfico No.2 Flujograma del Proceso de Comercio Exterior en el OBCE

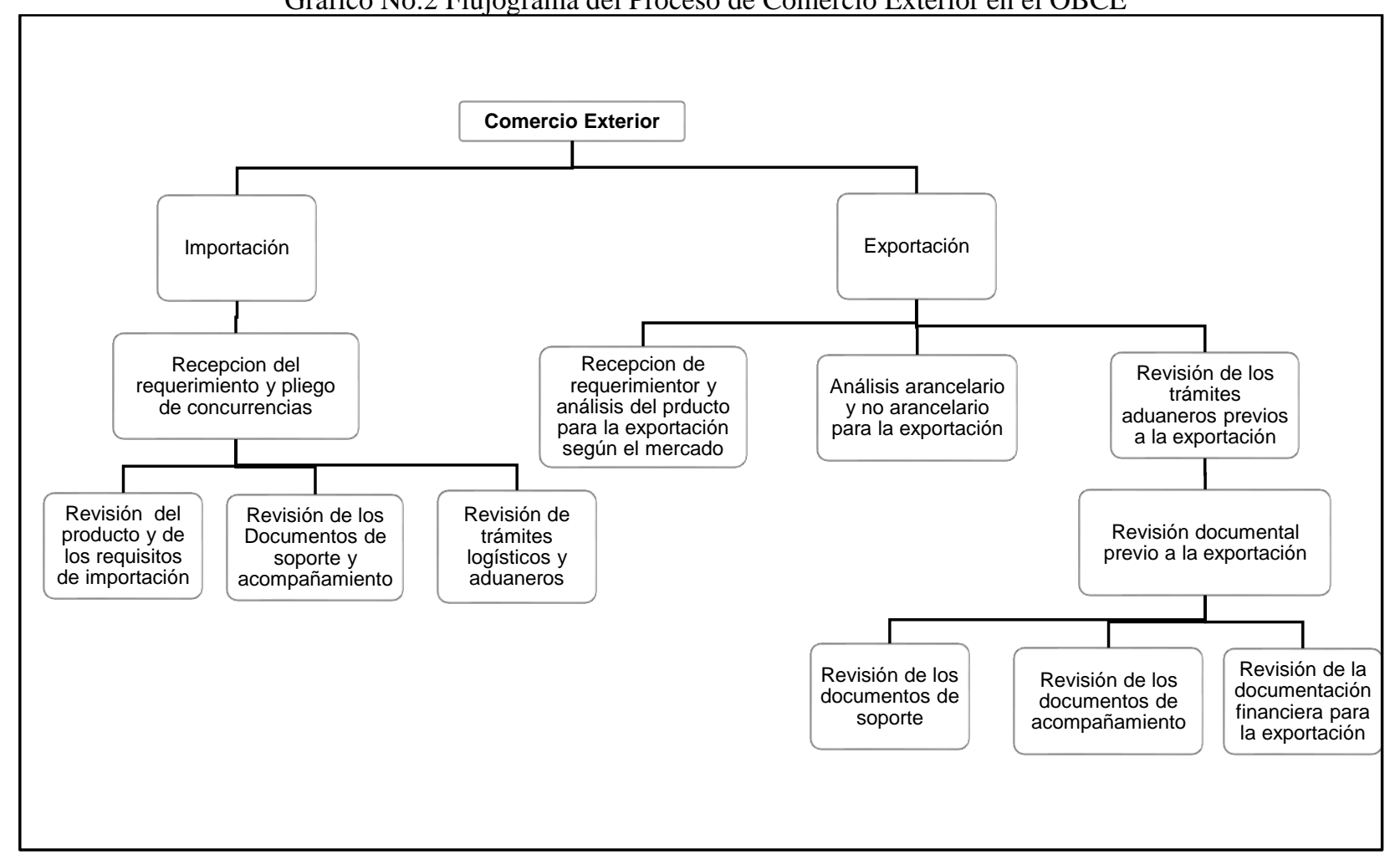

Elaborado por: Los autores

\subsection{RELACIONES INTERNACIONALES}

Las Relaciones Internacionales son vínculos que se establecen entre organizaciones o empresas que buscan tener acuerdos que los beneficie, por medio de políticas comerciales, acuerdos, solución de problemas, relaciones diplomáticas, optimizar las relaciones exteriores con miras a mejorar el comercio teniendo como fin vincularse con nuevos mercados, manteniendo la cooperación y buenas relaciones internacionales.

El Observatorio y las Relaciones Internacionales se adaptan para brindar información relevante sobre la relación económica comercial entre las regiones, así como por un ámbito en el cual se desarrollan actividades (foros, seminarios, etc.) que contribuyen a avanzar en el conocimiento y reflexión sobre esta relación estratégica, en colaboración con los países involucrados, organismos internacionales y regionales y mecanismos de integración que contribuyen a este proyecto (Observatorio América Latina, Asia Pacífico, 2015). A continuación, se presenta las actividades de los tres procedimientos vinculados a las relaciones internacionales del $\mathrm{OBCE}$, que son: investigación de programas de relaciones internacionales, contacto para la negociación, seguimiento al acuerdo al que se ha llegado (Ver gráfico 3). 
Gráfico No.3 Flujograma del Proceso de Relaciones Internacionales en el OBCE

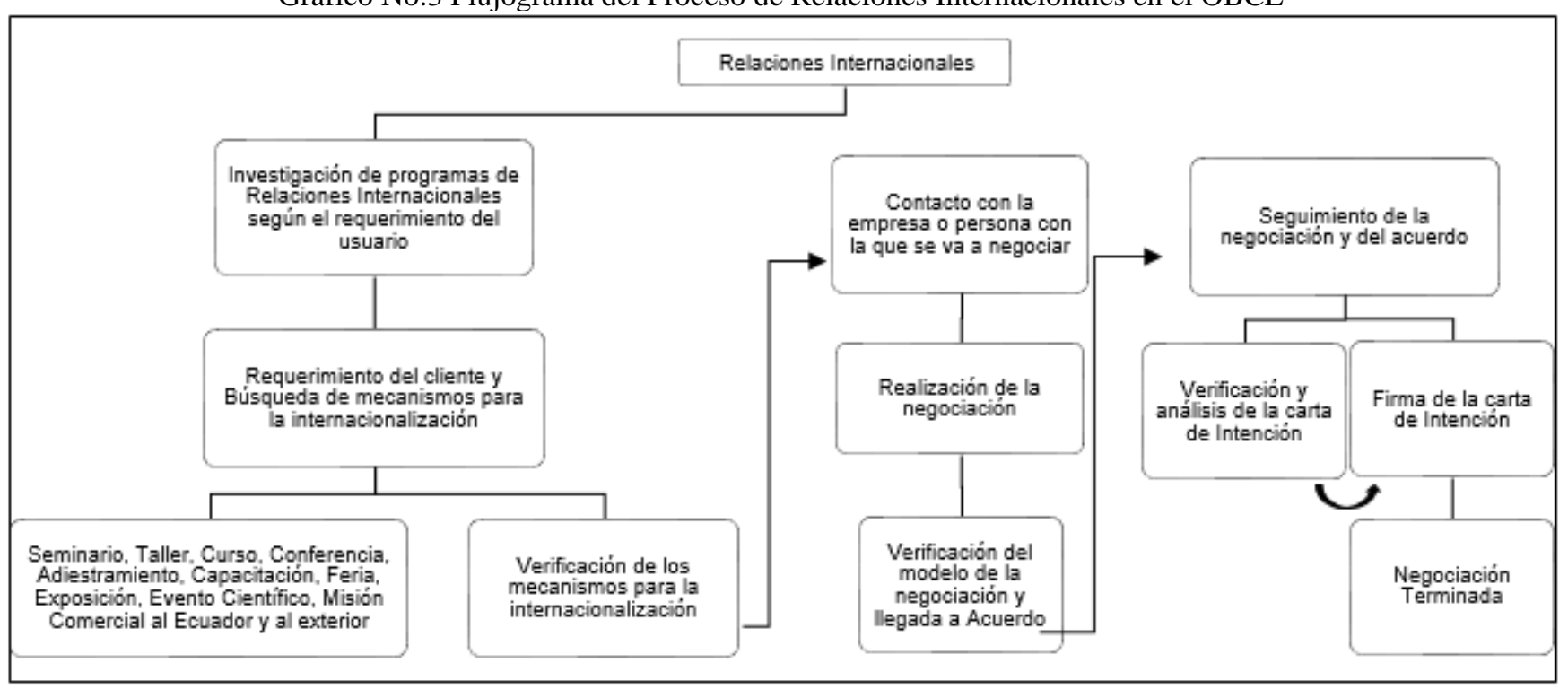

Elaborado por: Los autores

\subsection{INVERSIÓN EXTRANJERA}

Los factores para atraer la inversión extranjera directa (IED) a un país están relacionados a las ventajas competitivas en los procesos productivos, es decir los países con grandes reservas de recursos materiales o materias primas, tienen gran atractivo, además de aquellos que cuentan con fuerza de trabajo eficiente y capacitada, o bien que estén relacionados con mercados desarrollados.

El 90\% de IED mundial se moviliza entre los países más desarrollados, al haber logrado un alto desarrollo humano, social, industrial y tecnológico hace que la mayoría de empresas trabajen entre las mayores economías locales como Estados Unidos, Unión Europea o Japón. Esto se complementa con un incremento en la capacitación de los trabajadores locales por parte de las empresas extranjeras que invierten en el país. Los países en desarrollo como Ecuador tienen especial interés en estos flujos de capital porque son una excelente fuente de financiamiento para el desarrollo productivo de las actividades económicas. La IED en Ecuador se produce principalmente a través de multinacionales en construcción de nuevas plantas o la ampliación de sus instalaciones existentes en países extranjeros.

En este contexto, el proceso de inversión extranjera consta de cuatro procedimientos que apoyarán a la atracción de inversión extranjera a la zona norte del Ecuador: generación de información técnica y relevante sobre los flujos de inversión proveniente y saliente hacia mercados internacionales, identificación de mecanismo de atracción de inversión extranjera a partir de la investigación, canalización de la inversión extranjera, control y seguimientos de la utilización de los flujos de inversión extranjera (Ver gráfico 4). 
Gráfico No.4 Flujograma del Proceso de Inversión Extranjera en el OBCE

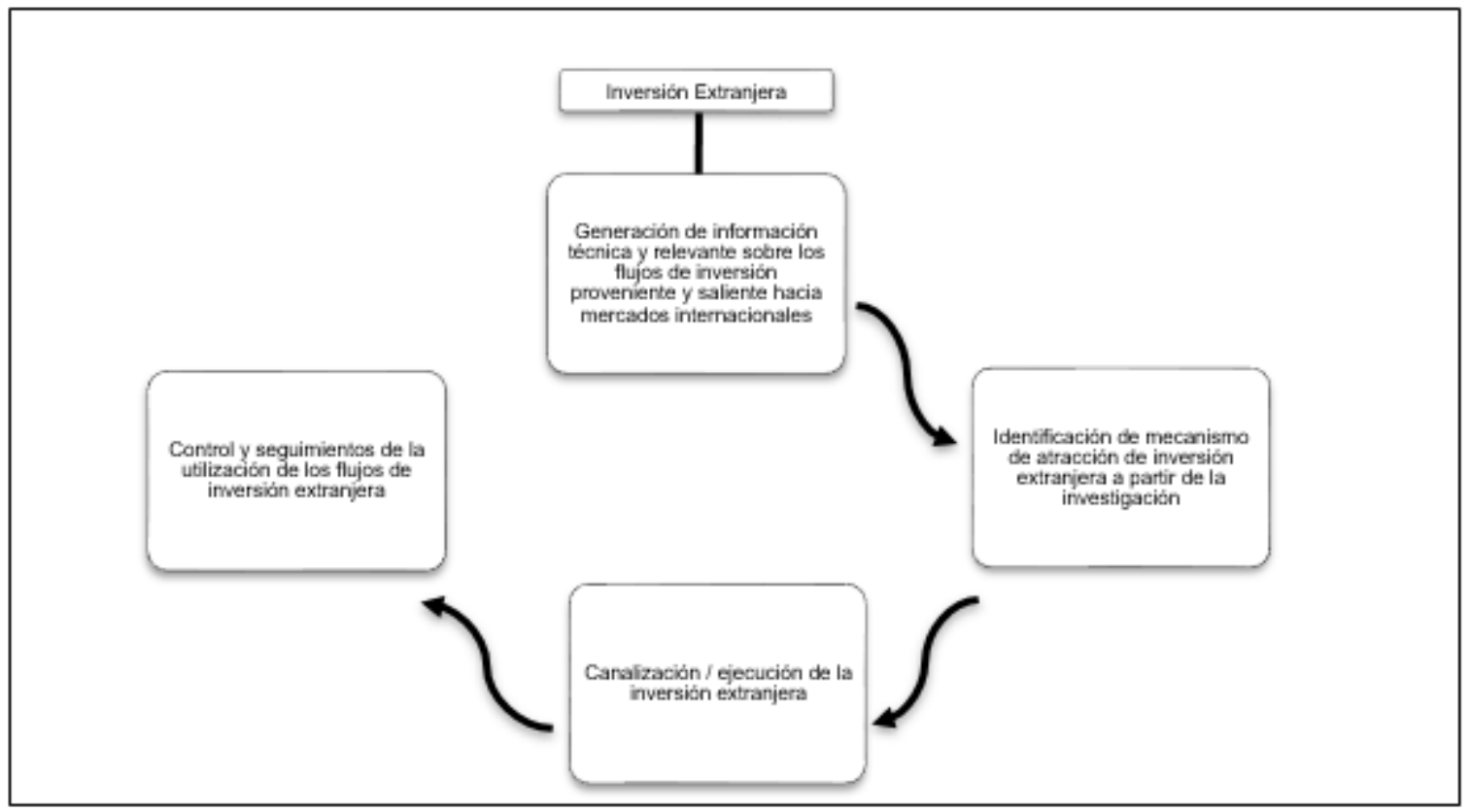

Elaborado por: Los autores

\section{CONCLUSIONES}

Se ha dado cumplimiento de forma satisfactoria al objetivo general del documento realizando una revisión detallada de las áreas que conforman la función operativa del Observatorio de Ciencias Empresariales de la Universidad de Otavalo.

El análisis realizado ha demostrado que los procesos de inteligencia comercial, comercio exterior, relaciones internacionales e inversión extranjera son los necesarios para cumplir con la razón de constitución del observatorio que corresponde a la generación de investigaciones en materia de comercio exterior e inversión extranjera y brindar asesoramiento en estos temas a las micro, pequeñas y medianas empresas de la zona norte del Ecuador en temas de comercialización internacional sin que eso implique que se pueda apoyar a través de programas u otras actividades al desarrollo del tejido empresarial ecuatoriano en torno al ámbito administrativo y financiero. Cabe señalar que los procesos operativos tratados en este artículo se enfocan en el comercio y los negocios internacionales, los cuales están en constante relación con las áreas funcionales del observatorio, que refieren al desarrollo e innovación de productos y servicios comercializables, y la promoción para su comercialización.

Los diferentes procesos que se han planteado en el documento sirven para investigar mercados internacionales, brindar asesoramiento en operaciones y documentos en comercio exterior, obtener los mecanismos necesarios para la canalización efectiva de inversión extranjera desde y hacia el Ecuador y colaborar en la negociación internacional para la apertura de nuevas alianzas estrategias para los usuarios del observatorio. 
El proceso de inteligencia comercial se enfoca en la generación de información tanto respecto del perfil del usuario como de las fichas técnicas de los productos y de los mercados internacionales. El proceso de comercio exterior realiza el acompañamiento al usuario en la importación y exportación de bienes y servicios. El proceso de relaciones internacionales abarca la identificación de actores internacionales que brinden ayuda a los usuarios del observatorio, la gestión de la negociación internacional con miras a un acuerdo de beneficio mutuo y el seguimiento a la carta de intención suscrita por las partes. El proceso de inversión extranjera genera flujos de capital que permiten el desarrollo productivo y económico de la zona norte del Ecuador a través de la identificación del mecanismo que más se ajuste a los requerimientos de cada usuario. 


\section{REFERENCIAS}

Álvarez, M. (2014). Manual para elaborar Manuales de Políticas y Procedimientos. Mexico D.F.: Panorama Editorial S.A.

Angulo, N. (2009). ¿Qué son los observatorios y cuáles son sus funciones? (I. P. Nacional, Ed.) Innovación educativa, 9 (47), 5-17.

Cano, J. L. (2015). La inteligencia de negocio como apoyo a la toma de decisiones en el ámbito académico. Revista Internacional de Gestión del Conocimiento y la Tecnología, 22. Recuperado de:

http://itemsweb.esade.edu/biblioteca/archivo/Business_Intelligence_competir_con_informacion.pdf

CONQUITO. (2017). CONQUITO. Recuperado de: http://www.conquito.org.ec/

Desrosière A. (1996). Reflejar o Instituir: La Invención De Los Indicadores Estadísticos, En: Metodología. Revista de Técnicas, métodos e instrumentos de investigación en Ciencias Humanas. No. 4, Bruselas, Bélgica.

Duque, A. (2013). ICESI. Recuperado de: https://repository.icesi.edu.co/biblioteca_digital/bitstream/10906/77010/1/propuesta_observatorio_come rcio.pdf

Hernández, R. Fernández C. Baptista P. (2010). Metodología de la investigación. 5ta Edición, pp. 1-656. México DF. México. McGraw-Hill.

Ortiz, L. (2008). Manual de Procesos y Procedimientos Bases Estratégica y Organizacionales. Cartagena: Hospital Universitario del Caribe.

Phélan C, M. (2007). La red observatorios locales de Barcelona. Fermentum, 17(18), 96-122. Recuperado de: http://www.redalyc.org/pdf/705/70504806.pdf

Real Academia. (2018). Real Academia. Obtenido de http://lema.rae.es/drae2001/srv/search?id=j5EQCr4GYDXX2r09FRW0.

UDENAR. (2010). Universidad de Nariño. Recuperado de: http://calidad.udenar.edu.co/wpcontent/uploads/2012/02/SGC-GU-01_Guia_Elaboracion_Control_Docs_SIGC.pdf

UNED. (2013). Observatorios de Comercio Exterior . Costa rica : UNED. 